\title{
Características fisiológicas e biomecânicas na corrida do triatlo: uma revisão narrativa
}

\author{
Physiological and biomechanical characteristics in \\ the triathlon race: a narrative review
}

Daniela Ongaratto, ${ }^{1}$ Rodrigo Gomes da Rosa, ${ }^{1}$ Flávio Antônio de Souza Castro, ${ }^{1}$ Adriana Marques Toigo, ${ }^{2}$ Leonardo Alexandre Peyré-Tartaruga'

'Universidade Federal do Rio Grande do Sul (UFRGS), Porto Alegre, RS, Brasil.

2 Centro Universitário La Salle, Canoas, RS, Brasil.

Recebido em: 15/11/2016 / Aceito em: 28/09/2017 / Publicado em: 01/10/2017

leotartaruga@gmail.com

\section{RESUMO}

O triatlo caracteriza-se por ser um esporte múltiplo composto por três modalidades: natação, ciclismo e corrida que ocorrem nessa ordem e em sequência. $\mathrm{Na}$ etapa da corrida, o atleta sofre efeitos decorrentes das modalidades que a antecederam (natação e ciclismo) podendo assim, alterar parâmetros biomecânicos e fisiológicos da corrida. Objetivo: revisar aspectos fisiológicos, bem como alterações na biomecânica da corrida em triatletas. Método: a busca foi realizada nas bases de dados: Scielo, Pubmed e Google Acadêmico. Resultados: foram incluídos na revisão 48 artigos e um livro. Considerações finais: a corrida de triatletas apresenta alterações na eficiência ventilatória, no $\mathrm{VO}_{2}$ e na cinemática angular, principalmente do joelho e tornozelo. Além disso, triatletas exibem uma maior inclinação do tronco contribuindo para padrões mais econômicos de movimento, especialmente em atletas altamente treinados.

Palavras-chave: Cinemática; Triatleta; Locomoção; Técnica de corrida; Atleta.

\section{ABSTRACT}

Triathlon is characterized as a complex sport composed by three modalities accordingly: swimming, cycling and running. In this context the swimming and cycling stages seem to influence some physiological and kinematic parameters in the running. Objective: the purpose of this study was to review physiological aspects as well as changes in the running biomechanics in triathletes. Method: a search was conducted in the database Scielo, Pubmed and Google Scholar. Results: Forty-eight articles and a book were included in the review. Closing remarks: the triathletes' running show changes on ventilatory efficiency, $\mathrm{VO}_{2}$, and kinematic changes, mainly in the knee and ankle. Furthermore, the triathletes exhibit a greater inclination of trunk, contributing for more economic movement patterns, especially in high-level athletes.

Keywords: Kinematics; Triathlete; Locomotion; Running technique; Athlete.

\section{INTRODUČ̃̃}

O triatlo é um esporte constituído por natação, ciclismo e corrida, as quais ocorrem nessa ordem e sequência. A modalidade tornou-se olímpica em 2000, em Sydney - Austrália. Essa competição serviu como ponto de partida e, desde então, o crescimento no número de praticantes deste esporte é contínuo, tornando-se assim, de grande importância pesquisas sobre as características biomecânicas e fisiológicas de cada etapa para a melhora no desempenho.

Para obter-se êxito neste esporte, o atleta necessita apresentar um desempenho uniforme nas três etapas da prova de triatlo, pois o resultado final é decorrente não só de uma modalidade. Portanto um bom desempenho nas três etapas (natação, ciclismo e corrida) é importante no triatlo, mas, além disso, a interação entre cada uma delas deve ser levada em conta para obter 
o melhor desempenho em uma prova. ${ }^{1-4}$ Ao observar a contribuição individual de cada etapa para o desempenho geral, entre os anos 1989 a 2013, na distância olímpica, os tempos parciais da natação e da corrida diminuíram ao longo dos anos e o tempo do ciclismo e o total permaneceu inalterada. Por outro lado, na distância de Ironman o tempo de ciclismo, da corrida e o tempo total diminuíram, enquanto o tempo da natação manteve-se estável. De maneira percentual observa-se que o ciclismo e a corrida representam respectivamente - 36\% e - $47 \%$ na distância olímpica, enquanto que para a distância de Ironman, ciclismo e corrida apresentaram contribuições semelhantes $-40 \%$ e a natação para ambas distâncias corresponde a - 18\% do total da prova. ${ }^{5}$

Nessa perspectiva, exigem-se no triatlo preparo físico e nível técnico aperfeiçoados em cada modalidade que o compõe. Além disso, os efeitos de uma modalidade sobre as outras podem interferir nos componentes biomecânicos e energéticos das mesmas, de forma a melhorar ou piorar o desempenho. De forma aguda sabemos que a natação não altera o desempenho do ciclismo ${ }^{6}$, com isso, as pesquisas têm avançado no sentido de avaliar a corrida após o ciclismo, ou seja, as duas etapas finais do triatlo. Acredita-se que a etapa da corrida é um elemento fundamental aos resultados finais da prova, principalmente nos minutos da etapa do ciclismo (transição ciclismo/corrida), devido aos ajustes posturais e de ativação dos músculos, podendo assim, afetar significativamente o resultado final da prova., ${ }^{7,8}$

Evidências indicam que possa haver alterações agudas fisiológicas na corrida após o ciclismo como: maior consumo de oxigênio $\left(\mathrm{VO}_{2}\right){ }^{9-13}$ pior economia de corrida em atletas de nível médio e melhora na economia de corrida em atletas de elite após o ciclismo. ${ }^{14} \mathrm{Um}$ dos aspectos importantes para os estudos envolvendo o triatlo é o nível de desempenho do atleta, pois diferentes respostas fisiológicas e biomecânicas são encontradas na literatura. Durante a corrida, o atleta adota um padrão de locomoção que requer menor custo energético (otimização entre o comprimento e frequência de passada)..$^{15} \mathrm{Em}$ contraste, a cadência auto selecionada durante o ciclismo não é a mais econômica, ${ }^{16}$ mas a mais eficiente..$^{17}$ Com isso, podemos notar diferenças entre as modalidades que compõem o triatlo.

Os efeitos cumulativos das modalidades que antecede a corrida são responsáveis pela desidratação, ${ }^{3,11,12}$ diminuição da atividade pulmonar, ${ }^{11,12,14}$ diminuição de glicogênio muscular ${ }^{3}$ e aumento da percentual do $\mathrm{VO}_{2}$ máximo, porém, ainda é possível perceber que existem lacunas teóricas e práticas a esse respeito, como as diferentes adaptações relacionadas ao nível de treinamento, respostas agudas e crônicas da corrida após o ciclismo. Sendo assim, uma revisão sobre estes aspectos auxilia na compreensão da mecânica e energética da corrida de triatletas, contribuindo para a construção do treinamento mais específico para a modalidade. Assim o objetivo deste estudo foi revisar do ponto de vista narrativo, aspectos fisiológicos, bem como alterações na biomecânica da corrida em triatletas. Além disso, o estudo procura realizar uma análise crítica sobre estas alterações, ressaltando resultados gerais sobre o tema e com indicações para futuros estudos sobre os aspectos biomecânicos e fisiológicos da corrida no triatlo.

\section{MÉTODO}

As pesquisas foram realizadas entre março a setembro de 2016. Foi utilizado o Portal Capes de banco de dados periódicos através das seguintes estruturas: Scielo, PubMed e Google Acadêmico. Os descritores utilizados foram definidos de acordo com as exigências de cada base de dados. Utilizouse os operadores lógicos and, or e and not para a busca dos artigos. Foram utilizadas as palavras "triathlon", "running", "fatigue", "biomechanics", "physiologic" e "injury", sem qualquer definição de sexo e/ou nível de treinamento. Os estudos foram limitados entre 1977 e 2016 . Foram revisados apenas os artigos nas versões completas publicadas.

\section{RESULTADOS E DISCUSSÕES}

Nesta revisão foram utilizados 48 artigos e um capítulo de livro, a partir destes, 28 itens compõem a revisão, delimitação e conceitos de biomecânica e parâmetros fisiológicos (quatorze deles foram relacionados a aspectos fisiológicos, oito aspectos biomecânicos envolvidos, e seis sobre aspectos neuromusculares). E, 21 foram em relação ao triatlo, nove foram relacionados com a transição ciclismo/corrida, três foram sobre lesões, quatro de corrida, quatro de ciclismo e um de natação no triatlo. A figura 1 apresenta o resumo do número de itens e áreas revisadas.

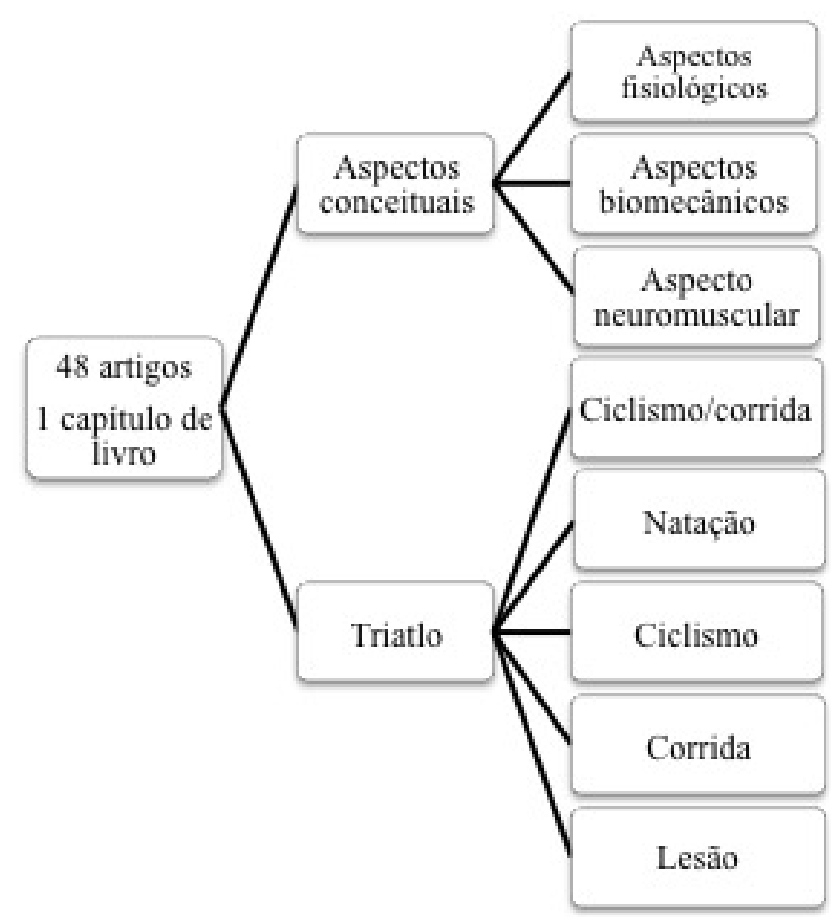

Figura 1 - Resumo do número de itens analisados e áreas analisadas e comparadas.

\section{Corrida no triatlo}

A corrida neste esporte é referida como uma etapa fundamental para o desempenho geral em uma prova, tendo em vista que seu resultado pode ser significativamente afetado pelas modalidades que a antecederam. ${ }^{9,11-13}$ 
Os efeitos da natação e do ciclismo na corrida posterior em provas de triatlo foram investigados em diferentes protocolos, o que ajuda a compreender aspectos fisiológicos e biomecânicos. Ainda, foram encontrados trabalhos que integram esses dois aspectos e merecem um olhar mais atento para entender de maneira mais integrativa os aspectos que permeiam a corrida, como o trabalho de Millet et al. ${ }^{14}$ que verificou alterações metabólicas e mecânicas na corrida após o ciclismo de intensidade máxima bem como os efeitos do nível de treinamento do atleta.

Ao se comparar a influência do ciclismo na corrida (transição ciclismo-corrida) com a corrida isolada, alguns autores revelaram que as modalidades prévias podem influenciar o desempenho do atleta na corrida. ${ }^{18-21}$ De acordo com os autores, isto tem sido atribuído, em função de pelo menos dois aspectos: alterações fisiológicas e alterações biomecânicas em relação à economia de movimento. A economia de movimento, pode ser definida como o custo de oxigênio para uma dada atividade submáxima. ${ }^{22}$

Entretanto, os estudos apresentam respostas diversas em relação a estes dois aspectos e o tipo de mudanças associadas a essas alterações, devido ao nível de treinamento e à variedade de distâncias em cada prova de triatlo, sendo estas as mais utilizadas, triatlo sprint ( $750 \mathrm{~m}$ natação - $20 \mathrm{~km}$ ciclismo - $5 \mathrm{~km}$ corrida), triatlo olímpico $(1500 \mathrm{~m}$ natação $-40 \mathrm{~km}$ ciclismo - 10 $\mathrm{km}$ corrida) entre outros, resultando assim, em diferentes resultados no desempenho do atleta na corrida durante o triatlo. ${ }^{23}$ Para exemplificar melhor tais respostas Bonacci et al. ${ }^{20}$ verificaram alterações biomecânicas no padrão da corrida subsequente ao ciclismo enquanto que nos estudos de Hue et al., ${ }^{18}$ Chapman et al. ${ }^{19} \mathrm{e}$ Fraga et al. ${ }^{21}$ não houve alterações nesse parâmetro.

\subsection{Alterações fisiológicas na corrida}

$\mathrm{Na}$ literatura, têm sido reportados alguns fatores relacionados às alterações fisiológicas na corrida do triatlo, sendo estes os mais citados:

Depleção dos estoques de glicogênio pelo ciclismo. ${ }^{14}$

2- Desidratação; ;,11,12

3- Diminuição da atividade pulmonar; ${ }^{11,12,14}$

4- Aumento do $\mathrm{VO}_{2}$ na corrida do triatlo e alterações na eficiência ventilatória; ${ }^{11,14,18,24}$

5- Elevada lactacidemia. ${ }^{9}$

Portanto é importante destacar que as alterações citadas acima, comumente, podem ter repercussão em ajustes cinemáticos, metabólicos e neuromusculares da corrida no triatlo.

Assim algumas variáveis intervenientes como, por exemplo, o comprimento e frequência de passada, o posicionamento do tronco, a angulação do joelho durante a fase aérea e extensão durante a fase de suporte interferem muitas vezes, no $\mathrm{VO}_{2}$, na eficiência ventilatória e no acréscimo do custo metabólico. ${ }^{25}$ Para Millet e Vleck, ${ }^{3}$ o aumento no custo metabólico varia de 1,6\% a $11,6 \%$ e isto é um reflexo do nível de habilidade do triatleta, ou seja, quanto maior a habilidade do atleta, menor é o aumento no custo metabólico.

Contudo, o trabalho mecânico, ligado diretamente com a eficiência de corrida, não é relacionado com a presença do componente lento da cinética do $\mathrm{VO}_{2}$ (aumento da taxa metabólica em condições de carga constante, geralmente presente em domínio metabólico pesado de exercício) em triatletas, o que indica que a energia mecânica despendida na corrida pode não estabelecer parâmetros que indiquem a queda no desempenho durante exercícios em domínio pesado ou severo de intensidade ${ }^{26}$ Neste sentido, Bonacci et al., ${ }^{20}$ relataram que a mudança na cinemática da angulação do joelho e tornozelo foram relacionadas ao $\mathrm{VO}_{2}$, quando os triatletas realizaram uma corrida submáxima depois do ciclismo. Estas modificações no ângulo do tornozelo, em relação ao contato do pé com o solo, explicaram $67,1 \%$ da variação no $\mathrm{VO}_{2}$ e estas alterações foram associadas à economia de movimento durante a corrida. De acordo com os autores, a associação entre o ângulo do tornozelo e $\mathrm{O} \mathrm{VO}_{2}$ foi positiva, com um aumento do ângulo do tornozelo (dorsiflexão) e isto foi associado a um aumento do $\mathrm{VO}_{2}$. De acordo com os autores, o aumento da dorsiflexão do tornozelo e extensão do joelho aumenta a força vertical de reação do solo e isto implica no custo metabólico, principalmente, durante uma corrida prolongada. Há uma tendência do calcanhar (retropé) reduzir a conversão de energia cinética de translação para energia cinética rotacional, uma vez que esta energia é desperdiçada no momento do contato com o solo. Por outro lado, uma maior flexão plantar melhora o desempenho do atleta devido à exploração do armazenamento e conversão de energia elástica. ${ }^{27}$

No estudo de Hue et al., ${ }^{18}$ foram avaliados os efeitos de $40 \mathrm{~km}$ de ciclismo (distância olímpica) nas variáveis cardiorrespiratórias em $10 \mathrm{~km}$ de corrida subsequente. Observaram que os primeiros minutos da corrida do triatlo (transição ciclismo-corrida) apresentaram respostas fisiológicas específicas para variáveis metabólicas e cardiorrespiratórias, como por exemplo, maiores valores para o $\mathrm{VO}_{2}$ (especialmente durante os 7 minutos de corrida), ventilação pulmonar, equivalentes respiratórios para oxigênio $\left(\mathrm{O}_{2}\right)$ e dióxido de carbono $\left(\mathrm{CO}_{2}\right)$, frequência cardíaca quando comparada com a corrida isolada. Entretanto, o tempo necessário para atingir o estado estável foi menor na frequência cardíaca e $\mathrm{VO}_{2}(1 \mathrm{~min}$. e $2 \mathrm{~min}$. respectivos) e maior para a ventilação-minuto (VE, 7min.).

Alguns estudos têm verificado aumento no $\mathrm{VO}_{2}$, na ventilação pulmonar, na diferença arteriovenosa de $\mathrm{O}_{2}$ e na frequência cardíaca durante a corrida precedida de ciclismo. ${ }^{18,28,29}$ Para explicar este aumento no $\mathrm{VO}_{2}$, alguns autores atribuem, em parte, à depleção do glicogênio durante os $40 \mathrm{~km}$ de ciclismo, concomitante com a alteração na utilização de substrato, no qual há uma predominância na mobilização para oxidação de gordura.$^{18}$ Por outro lado, acredita-se que este aumento tem relação parcial com a desidratação $0^{11,12,14}$ atribuindo com a hemoconcentração, diminuição do volume sistólico, aumento na temperatura retal após o ciclismo ${ }^{28}$ e a perda de peso corporal durante a corrida. ${ }^{28,29}$ Contudo a concentração de lactato não teve alteração na condição de corrida precedida de ciclismo. ${ }^{29}$

Hue et al. ${ }^{30}$ avaliaram as respostas ventilatórias na transição ciclismo/corrida, o qual concluiu que esta transição induz alterações na função pulmonar, como por exemplo, no aumento do volume residual, na capaci- 
dade funcional residual e na relação do volume residual com a capacidade total pulmonar e diminuição na capacidade de difusão pulmonar para o dióxido de carbono. Especula-se que estas alterações estejam associadas à fadiga muscular respiratória e/ou a hipoxemia induzida pelo exercício. De acordo com estes dados, é sugerido que o aumento na resposta ventilatória, principalmente nos primeiros minutos da corrida após o ciclismo, é devido à especificidade da modalidade e isto pode implicar na redução do desempenho durante a corrida. Portanto isto reforça a necessidade dos triatletas terem de treinar as transições, como por exemplo, transição do ciclismo para corrida, podendo assim, se adaptar as demandas fisiológicas essenciais para o desempenho do atleta em atividades subsequentes.

Utilizando um delineamento experimental, o qual foi investigado a economia de movimento no final de um triatlo simulado, a $60 \% \mathrm{VO}_{2}$ máximo. (6 atletas de nível inferior vs 6 de nível superior), verificou-se que os atletas de nível inferior apresentaram um aumento significativo desde os primeiros minutos de corrida no $\mathrm{VO}_{2}$ VE e frequência cardíaca, comparados com os atletas de nível superior. ${ }^{31}$ Peiffer et al. ${ }^{32}$ avaliaram a eficiência do ciclismo e o custo metabólico da corrida em triatletas jovens $(n=20)$ e másters $(n=20)$ durante um teste de 10 min em velocidade submáxima de corrida e um teste de $10 \mathrm{~min}$ de ciclismo na intensidade do primeiro limiar ventilatório. A eficiência no ciclismo foi menor ($11,2 \%)$ nos triatletas másters comparado aos jovens, e o custo metabólico da corrida foi maior nos másters (+ $10,8 \%)$. Demonstrando assim que, em triatletas treinados, o envelhecimento pode influenciar no desempenho metabólico tanto no ciclismo quanto na corrida.

Embora geralmente não sejam encontradas alterações no ciclismo precedido de natação, no estudo de Costa e Kokubun ${ }^{9}$ foi observada uma diminuição do desempenho no ciclismo ocorrida após a natação. Provavelmente, os triatletas encontravam-se em acidose, pois a velocidade do nado foi acima do ritmo de limiar de Lactato. Portanto sabe-se que, a concentração de lactato alta durante a natação pode afetar negativamente o desempenho da atividade subsequente. ${ }^{33}$

Do mesmo modo, a alteração na corrida do triatleta após o ciclismo é relacionada à carga metabólica sustentada até o final do ciclismo com um aumento de concentração de lactato sanguíneo, ${ }^{9,12}$ atribuído à acidose antes do início da próxima etapa. Del Coso et al. ${ }^{34}$ avaliaram variáveis fisiológicas e o desempenho em uma prova de triatlo meio-ironman realizada em alta temperatura ambiente $\left(29 \pm 3^{\circ} \mathrm{C}\right)$. Trinta e quatro triatletas bem treinados completaram a prova e, antes e após a prova, foram coletados valores de massa corporal, temperatura central, a altura de salto vertical e amostras de sangue venoso. O tempo médio de prova foi de 315 $\pm 40 \mathrm{~min}$, com as parciais de tempo de natação de $11 \%( \pm 1 \%)$, ciclismo de $49 \%( \pm 2 \%)$ e corrida de 40 $\%( \pm 3 \%)$ que representam diferentes quantidades do tempo total de prova. No final da competição, a massa corporal foi alterada em - 3,8 $\pm 1,6 \%$ e a mudança na massa corporal foi positivamente correlacionada com o tempo de corrida $(r=0,64 ; P<0,001)$. A temperatura dos atletas aumentou de $37,5 \pm 0,6^{\circ} \mathrm{C}$ para $38,8 \pm$ $0,7^{\circ} \mathrm{C}(\mathrm{P}<0,001)$ e pós-corrida a temperatura central teve correlação negativa com o tempo de corrida $(r=-$ $0,47 ; P=0,007)$. O tempo de corrida foi positivamente correlacionado com a diminuição da altura do salto ( $r$ $=0,38 ; P=0,043$ ) com os níveis séricos de creatina quinase ( $r=0,55 ; P=0,001)$ e com a concentração de mioglobina $(r=0,39 ; P=0,022)$. As maiores reduções na massa corporal e temperatura central mais elevadas após a prova estavam presentes em triatletas mais rápidos. Em contraste, triatletas mais lentos apresentaram maiores níveis de lesão muscular e diminuição do desempenho muscular.

\subsection{Alterações na biomecânica da corrida}

Dentro do triatlo, a etapa da corrida implica ao triatleta modificações neuromusculares, principalmente nos primeiros minutos subsequentes ao ciclismo (transição ciclismo-corrida).7,8 Essas alterações ocorrem, principalmente devido ao efeito cumulativo nas etapas que a antecederam, contribuindo para um decréscimo no desempenho e assim no desempenho geral durante a prova. ${ }^{18,35}$ Neste sentido, compreender a biomecânica da corrida é um importante aspecto para melhorar a execução da técnica, e assim seu desempenho, com a implementação de ferramentas que auxiliem no treinamento. Apesar de ser uma forma de locomoção natural, ao mesmo tempo é complexa, pois está relacionada com a necessidade da coordenação do movimento, ${ }^{36}$ economia neuromuscular, ${ }^{37}$ e eficiência mecânica, ${ }^{38}$ entre outros aspectos.

Foram observados prejuízos na economia de movimento de corrida após o ciclismo $8,14,20$ (Tabela 1). Estes prejuízos estão relacionados com alterações mecânicas como amplitude angular do joelho e tornozelo causados por meio de uma maior inclinação do tronco para frente.

Os parâmetros do comprimento e frequência de passada mostraram-se relativamente constantes na corrida após o ciclismo. ${ }^{13}$ Dados que corroboram com os resultados de Hue et al., ${ }^{18}$ no qual frequência e comprimento de passada permaneceram inalterados durante a corrida após o ciclismo na distância olímpica $(40 \mathrm{~km}$ ciclismo e $10 \mathrm{~km}$ corrida). Portanto supõe-se que o ciclismo não altera os padrões da passada na corrida. ${ }^{18,39}$ Porém, mudanças no desempenho do atleta como, por exemplo, o aumento da frequência de passada e uma diminuição no comprimento de passada para manutenção da velocidade constante na corrida, estão, muitas vezes, relacionadas à fadiga muscular e/ou alterações na atividade muscular, e, além disto, provavelmente contribuem para alterações na variação de potência muscular na corrida. ${ }^{7,10-12,14}$

A cinemática da corrida não é influenciada por períodos curtos e de intensidade moderada no ciclismo em atletas altamente treinados, além de não apresentar diferenças na ativação do músculo tibial anterior, porém, é importante destacar que, no estudo de Chapman e colaboradores ${ }^{19}$ o recrutamento muscular do músculo tibial anterior, durante a fase de apoio da corrida, foi diferente em alguns atletas apesar dos anos de treinamento. Tal fato foi associado ao efeito direto do ciclismo no padrão de comando motor e não apenas aos efeitos da fadiga, que estão mais relacionados com atividade muscular.

O músculo tibial anterior tem um papel primordial no controle da posição da articulação do tornozelo e 
da regulação da força da perna. Portanto a mudança na ativação do músculo tibial anterior pode alterar o desempenho e efeito de carga tanto no tecido mole quanto no tecido ósseo. A sobrecarga pode ocasionar micro fraturas ao tecido ósseo implicando muitas vezes em lesões por overuse (por exemplo, fratura por estresse)..$^{19}$ Esta lesão é muito comum em corredores acometidos nos membros inferiores. ${ }^{40-43}$

As alterações no recrutamento muscular contribuem para a modulação da rigidez do membro inferior e, por conseguinte, com a utilização da energia elástica armazenada. ${ }^{44}$ Durante $10 \mathrm{~km}$ de corrida, em uma prova de triatlo, a rigidez de membros inferiores e a rigidez vertical não se modificaram ao longo da prova, indicando que o sistema massa-mola não foi alterado. ${ }^{45}$

O desempenho da corrida no triatlo parece ter relação com a cadência empregada na etapa de ciclismo durante o triatlo, visto que em estudos realizados em laboratório, a cadência baixa no ciclismo $(73$ rpm - rotação por minuto) reduz a captação de oxigênio durante a transição ciclismo-corrida, quando comparada com a seleção de cadências elevadas (80-90 rpm). ${ }^{11}$ Os autores sugerem que a escolha da cadência baixa $(<80 \mathrm{rpm})$ antes da transição pode ter benefícios para o desempenho do atleta na corrida subsequente. Enquanto que a escolha da cadência elevada implica em um aumento no custo de energia durante o ciclismo com recrutamento maior de fibras do tipo II e consequentemente, contribui para o surgimento do componente lento de $\mathrm{VO}_{2}$ durante a corrida subsequente.

No entanto, em estudos de campo, o uso de cadências elevadas no ciclismo resultou em um melhor desempenho em teste de $3200 \mathrm{~m}$ realizado em pista, do que com cadências mais baixas (70-90 rpm), para um grupo de triatletas treinados. Portanto sugere-se que a seleção da cadência mais elevada no ciclismo melhora o desempenho do atleta na corrida com aumento da frequência de passada, resultando assim, em aumento da velocidade média durante a corrida. ${ }^{46}$

Por outro lado, ao comparar o efeito de diferentes cadências (60-100 rpm) de ciclismo no desempenho da corrida nos $3000 \mathrm{~m}$ não foram encontradas diferenças entre elas, contudo a escolha de $60 \mathrm{rpm}$ foi associada com uma fração mais elevada de $\mathrm{VO}_{2}$ máximo sustentada durante a corrida em comparação com as outras condições. Os autores acreditam que há uma maior contribuição do sistema aeróbio em cadências mais baixas, que podem assim, retardar a fadiga por mais tempo na etapa da corrida. ${ }^{12}$

Mudanças na ativação dos músculos do tronco podem ser devidas à modificação na contração muscular abdominal e lombar na transição entre o ciclismo e a corrida. ${ }^{14}$ Uma maior coativação muscular devido à mudança de posição pode prejudicar a economia neuromuscular, tendo um custo energético maior para atingir padrões de movimento diferentes entre o ciclismo e a corrida. Atletas mais treinados apresentam maior economia de movimento e, consequentemente, menor prejuízo no desempenho quando comparados com atletas menos experientes com a modalidade. ${ }^{37}$

De acordo com esta comparação, atletas de nível médio apresentam um aumento no deslocamento vertical do centro de massa na fase inicial de contato do pé com o solo na corrida nos primeiros minutos de corrida, um maior $\mathrm{VO}_{2}$ e alterações na mecânica da corrida após o ciclismo quando comparados com atletas de elite. ${ }^{14}$

Tabela 1 - Estudos analisados metodologicamente para comparação sistemática.

\begin{tabular}{|c|c|c|c|c|c|c|}
\hline AUTOR & AMOSTRA & METODOLOGIA & $\begin{array}{l}\text { PROTOCOLO } \\
\text { CICLISMO }\end{array}$ & $\begin{array}{l}\text { PROTOCOLO } \\
\text { CORRIDA }\end{array}$ & RESULTADOS & COMENTÁRIOS \\
\hline $\begin{array}{l}\text { Millet e Vleck, } \\
2000^{3}\end{array}$ & & Revisão & & & $\begin{array}{l}\text { Aumento na inclinação } \\
\text { pra frente do tronco. }\end{array}$ & $\begin{array}{l}\text { Devido à contração muscular ab- } \\
\text { dominal e lombar, induzidas pelas } \\
\text { mudanças da posição do corpo } \\
\text { entre o ciclismo e a corrida. }\end{array}$ \\
\hline $\begin{array}{l}\text { Vercruyssen } \\
\text { et al., } 2002^{11}\end{array}$ & 8 atletas & 3D & $\begin{array}{c}30 \text { minutos } \\
\text { Valores do limiar } \\
\text { ventilatório }+5 \%\end{array}$ & $\begin{array}{l}15 \text { minutos } \\
85 \% \text { da } \\
\text { velocidade } \\
\text { máxima }\end{array}$ & $\begin{array}{l}\text { Aumento FP; } \\
\text { Diminuição CP }\end{array}$ & $\begin{array}{l}\text { Mudanças no recrutamento mus- } \\
\text { cular ou modificações hemod- } \\
\text { inâmicas durante o exercício } \\
\text { prolongado. }\end{array}$ \\
\hline $\begin{array}{l}\text { Fraga et al., } \\
2006^{13}\end{array}$ & $\begin{array}{l}09 \text { atletas mascu- } \\
\text { linos. Idade média: } \\
\quad 32 \text { anos. }\end{array}$ & $\begin{array}{l}2 \mathrm{D} \\
\mathrm{EMG}\end{array}$ & $40 \mathrm{~km}$ & 10 km & $\begin{array}{l}\text { Aumento no valor RMS do } \\
\text { músculo vasto lateral }\end{array}$ & $\begin{array}{l}\text { Aumento relacionado ao pro- } \\
\text { cesso de fadiga muscular. }\end{array}$ \\
\hline $\begin{array}{l}\text { Chapman et } \\
\text { al., } 2008^{19}\end{array}$ & $\begin{array}{l}16 \text { atletas de Elite. } \\
\text { Idade média: } \\
\text { 30,2 anos. }\end{array}$ & $\begin{array}{c}2 D \\
3 D \\
\text { EMG }\end{array}$ & 20 minutos & 30 minutos & $\begin{array}{l}\text { Alteração na atividade mus- } \\
\text { cular TA em } 46 \% \text { dos atletas. }\end{array}$ & $\begin{array}{l}\text { Esta alteração esta associada ao } \\
\text { efeito do ciclismo. }\end{array}$ \\
\hline $\begin{array}{l}\text { Bonacci et al., } \\
2010^{20}\end{array}$ & 15 atletas & $3 D$ & $\begin{array}{l}\text { 45minutos } \\
\text { intensidade alta }\end{array}$ & Submáxima & $\begin{array}{l}\text { Mudanças na cinemática } \\
\text { do joelho e tornozelo. } \\
\text { Alterações no recrutamento } \\
\text { muscular nos membros } \\
\text { inferiores. }\end{array}$ & $\begin{array}{l}\text { Cinemática alterada em } 46 \% \\
\text { dos atletas. As quais foram as- } \\
\text { sociadas às mudanças no } \mathrm{VO}_{2} \text {. }\end{array}$ \\
\hline $\begin{array}{l}\text { Rendos et al., } \\
2013^{8}\end{array}$ & $\begin{array}{l}28 \text { atletas. } \\
\text { Idade média: } \\
24.6 \text { anos. }\end{array}$ & $\begin{array}{l}2 D \\
3 D\end{array}$ & 30minutos & 14 minutos & $\begin{array}{l}\text { Alterações na: Coluna } \\
\text { Vertebral, } \\
\text { Cintura Pélvica e Quadril. }\end{array}$ & $\begin{array}{l}\text { Pedalar na posição aerodinâmica } \\
\text { pode induzir alterações na } \\
\text { cinemática durante a corrida. } \\
\text { Estas alterações podem poten- } \\
\text { cializar lesões. }\end{array}$ \\
\hline
\end{tabular}

Nota: EMG Eletromiografia. RMS (root mean square: valor indicativo do nível de ativação muscular, expresso em percentual do pico). FP frequência de passada e CP comprimento de passada. TA tibial anterior. 
Millet et al. ${ }^{14}$ observaram que houve uma diminuição de $3,7 \%$ do $\mathrm{VO}_{2}$ em triatletas de elite e um aumento deste parâmetro para triatletas de nível regional sendo de 2,3\% após o ciclismo. Entretanto este parâmetro não teve diferença em atletas das categorias júnior e sênior de elite do sexo masculino, mas houve um aumento no $\mathrm{VO}_{2}$ em triatletas da categoria júnior do sexo feminino do que as da categoria sênior de elite. ${ }^{47}$

Supõe-se que este aumento no deslocamento do centro de massa possa alterar as características de impacto e de força na corrida. ${ }^{48}$ Quando este deslocamento do centro de massa é excessivo atribui-se a uma técnica ineficiente, ocasionando um aumento do custo energético para a projeção vertical do corpo. ${ }^{13}$ Atletas de elite têm uma melhor regulação da rigidez do membro inferior quando comparados com atletas de nível médio. ${ }^{14}$

Portanto a adaptação neuromuscular durante a corrida (após o ciclismo) varia conforme o estado de treinamento, ou seja, atletas altamente treinados exibem padrões de recrutamento muscular mais refinados e o gesto motor mais adequado do que os atletas novatos ${ }^{19}$ o que repercute em uma maior economia de movimento contribuindo para otimização do desempenho durante a prova (e talvez, diminuindo os riscos de lesões musculares decorrentes de quedas ou assimetrias do movimento).

Alguns atletas iniciantes relatam perda de coordenação dos membros inferiores na transição (ciclismo-corrida). Efeitos desta alteração na coordenação são em grande parte desconhecidos. ${ }^{44}$ Para alguns autores este fenômeno refere-se à mudança da intensidade no ciclismo e/ ou a mudança de atividade do músculo por não suportar as forças de impacto de 2-3 vezes o peso corporal em cada passada, ${ }^{14}$ enquanto que o mesmo não ocorre no ciclismo. A contração muscular durante o ciclismo é diferente da corrida, o qual é caracterizado por mais tempo em fases de contração muscular concêntrica, enquanto que na corrida envolve etapas sucessivas de contração muscular excêntrico-concêntrico. ${ }^{7}$

De acordo com Rendos et al., 8 pedalar em uma posição aerodinâmica (quando o atleta se posiciona em uma flexão prolongada do tronco com as mãos no guidão) por 30 minutos induz mudanças no plano sagital da cinemática da corrida. E tem se mostrado indutora de deformação viscoelástica dos músculos paraespinhais. Estes autores observaram alterações na coluna vertebral, pélvica e quadril durante 14 minutos após o ciclismo. Alterações na cinemática podem aumentar o risco de lesão nos membros inferiores e afetar o desempenho na corrida em triatletas. Contudo, o posicionamento do atleta mais inclinado na bicicleta pode trazer benefícios para a corrida, uma vez que o músculo gastrocnêmio e o bíceps femoral geralmente são menos ativados, e pode ocorrer uma menor fadiga dessa musculatura, permitindo assim, um aumento do comprimento de passada e eficiência durante a corrida. ${ }^{49}$

Embora os aspectos biomecânicos e fisiológicos da corrida durante provas de triatlo estejam bem descritos, como observado anteriormente, algumas limitações gerais podem ser observadas nestes estudos. Um aspecto limitante que deve ser endereçado em estudos futuros é sobre o modo de expressão da economia de corrida, so- bre o uso do custo de oxigênio, ou do custo energético, ou seja tendo em conta sobre o substrato metabolizado, este último mais recomendado. ${ }^{50}$ Outra questão é sobre a necessidade de análise individual com o uso de tamanho de efeitos por triatleta, e a utilização de grupos ou situações controle para vários estudos que tratam do tema. ${ }^{51}$ Outra observação advinda da análise geral do artigo é de que do ponto de vista metodológico, os estudos não se modificaram substancialmente dos anos 2000 até os dias atuais. Os métodos para avaliação das determinantes fisiológicas e biomecânicas do desempenho não se modificaram, e este ponto abre um campo de novas possibilidades de análise para a corrida no triatlo. Por exemplo os avanços nos métodos de análise biomecânicos, como da determinação das assimetrias contato-despregue ${ }^{52,53}$ e da determinação da eficiência mecânica, tendo em consideração a contra-parte anaeróbica no seu denominador, ${ }^{54}$ e o trabalho mecânico interno no seu numerador. ${ }^{55}$ Estas novas possibilidades podem melhorar a análise dos fatores determinantes fisiológicos e biomecânicos da corrida do triatlo.

\section{CONSIDERACÕES FINAIS}

A corrida no triatlo é referida como um elemento fundamental para a conclusão da prova, tendo em vista que o desempenho do atleta durante esta fase pode ser afetado pelas modalidades prévias (natação e ciclismo) tanto nos parâmetros fisiológicos bem como biomecânicos, porém ainda há muita controvérsia sobre os efeitos da mesma durante a corrida. Além disso, é importante destacar que há uma variedade de distâncias e diferentes formatos de provas de triatlo com diferentes alterações mecânicas e metabólicas que podem modificar o desempenho do atleta durante a corrida no triatlo.

$\mathrm{Na}$ corrida do triatlo, há uma diminuição do comprimento de passada e alterações angulares no joelho, tornozelo e no tronco atribuídos aos efeitos do ciclismo. Estas modificações parecem estar relacionadas à fadiga e/ou alterações na atividade muscular, podendo assim, prejudicar o desempenho do atleta no restante da prova, especialmente na etapa da corrida. Em contraste, não foi observado alterações nestes parâmetros em alguns estudos. Percebe-se que atletas altamente treinados não sofrem com estas alterações, portanto supõe-se que estes triatletas estão mais adaptados com a transição natação/ciclismo e ciclismo/corrida, inclusive apresentando maior economia de movimento durante a corrida com consequente melhora no desempenho. Por isso, acredita-se que é útil o treinamento de transições, especialmente a transição ciclismo/corrida, uma vez que os atletas possam treinar em um cenário mais próximo da prova, respeitando assim, a especificidade de treinamento da modalidade.

Embora existam recomendações de treinamento baseadas em achados apresentados nesta revisão, são necessários mais estudos, que analisem parâmetros fisiológicos e biomecânicos e suas adaptações de longo prazo acerca dos efeitos da transição ciclismo/corrida, em estudos longitudinais. 


\section{REFERÊNCIAS}

1. O'Toole ML, Douglas PS, Hiller WD. Applied physiology of a triathlon. Sports Med 1989;8(4):201-25.

2. Margaritis I. Facteurs limitants de la performance en triathlon. Canadian Journal of Applied Physiology 1996;21(1):1-15. doi: 10.1139/h96-001.

3. Millet GP, Vleck V. Physiological and biomechanical adaptations to the cycle to run transition in olympic triathlon: review and practical recommendations for training. $\mathrm{Br} \mathrm{J}$ Sports Med 2000;34(5):384-90. doi: 10.1136/bjsm.34.5.384

4. Diefenthaeler F, Candotti CT, Ribeiro J. Oliveira AR. Comparação de respostas fisiológicas absolutas e relativas entre ciclistas e triatletas. Rev Bras Med Esporte 2007; 13(3):205-8. doi: 10.1590/S1517-86922007000300015

5. Figueiredo $P$, Marques EA, Lepers $R$. Changes in Contributions of Swim, Cycle, and Run Performances on Overall Triathlon Performance over a 26-year period. Journal of Strength and Conditioning Research. 2016. doi: 10.1519/ JSC.0000000000001335

6. Laursen PB, Rhodes EC, Langill RH. The effects of 3000$\mathrm{m}$ swimming on subsequent $3-\mathrm{h}$ cycling performance: implications for ultra-endurance triathletes. Eur J Appl Physiol 2000;83(1):28-33. doi: 10.1007/s004210000229

7. Vercruyssen F, Suriano R, Bishop D, Hausswirth C, Brisswalter J. Cadence selection affects metabolic responses during cycling and subsequent running time to fatigue. $\mathrm{Br} \mathrm{J}$ Sports Med 2005;39(5):267-72. doi: 10.1136/bjsm.2004.011668

8. Rendos NK, Harrison BC, Dicharry JM, Sauer LD, Hart JM. Sagittal plane kinematics during the transition run in triathletes. J Sci Med Sport 2013;16(3):259-65. doi: 10.1016/j.jsams.2012.06.007

9. Costa JM, Kokubun E. Lactato sanguíneo em provas combinadas e isoladas do Triatlo: possíveis implicações para o desempenho. Ver Paul Educ Fís 1995;9(2):125-30.

10. Gottschall JS, Palmer BM. Acute effects of cycling on running step length and step frequency. Journal of Strength and Conditioning Research 2000;14(1):97-101.

11. Vercruyssen F, Brisswalter J, Hausswirth C, Bernard T, Bernard O, Vallier JM. Influence of cycling cadence on subsequent running performance in triathletes. Med Sci Sports Exerc 2002;34(3):530-6.

12. Bernard T, Vercruyssen F, Grego F, Hausswirth C, Lepers $\mathrm{R}$, Vallier JM et al. Effect of cycling cadence on subsequent $3 \mathrm{~km}$ running performance in well trained triathletes. $\mathrm{Br} J$ Sports Med 2003;37(2):154-8. doi: 10.1136/bjsm.37.2.154

13. Fraga CHW, Bloedow LLS, Carpes F, Tartaruga LAP, Follmer B, et al. Proposta metodológica para análise cinemática e fisiológica da corrida no Triathlon. Rev Mot 2006;12(2):159-164.

14. Millet GP, Millet GY, Hofmann MD, Candau RB. Alterations in running economy and mechanics after maximal cycling in triathletes: influence of performance level. Int J Sports Med. 2000;21(2):127-32.

15. Cavanagh PR, Williams, KR. The effect of stride length variation on oxygen uptake during distance running. Med Sci Sports Exerc 1982;14(1):30-5.

16. Marsh AP, Martin PE. Effect of cycling experience, aerobic power, and power output on preferred and most economical cycling cadences. Med Sci Sports Exerc 1997;29(9):122532. doi: 10.1097/00005768-199709000-00016

17. Samozino P, Horvais N, Hintzy F. Interactions between cadence and power output effects on mechanical efficiency during sub maximal cycling exercises. Eur J Appl Physiol 2006;97(1):133-9. doi 10.1007/s00421-006-0132-x
18. Hue O, Le Gallais D, Chollet D, Boussana A, Préfaut C. The influence of prior cycling on biomechanical and cardiorespiratory response profiles during running in triathletes. Eur J Appl Physiol Occup Physiol 1998;77(12):98-105. doi: $10.1007 / \mathrm{s} 004210050306$

19. Chapman AR, Vicenzino B, Blanch P, Dowlan S, Hodges PW. Does cycling effect motor coordination of the leg during running in elite triathletes? J Sci Med Sport 2008;11(4):37180. doi: 10.1016/j.jsams.2007.02.008

20. Bonacci J, Green D, Saunders PU, Blanch P, Franettovich $M$, Chapman AR, et al. Change in running kinematics after cycling are related to alterations in running economy in triathletes. J Sci Med Sport 2010;13(4):460-4. doi: 10.1016/j.jsams.2010.02.002

21. Fraga $\mathrm{CHW}$, Bianco $\mathrm{R}$, Serrão JC, Souza PES, Amadio ACA, Guimarães ACS, et al. Comparação das variáveis eletromiográficas e cinemáticas entre uma corrida do "triathlon" euma corrida prolongada. Rev Bras Educ Fís Esporte 2013;27(2). doi: 10.1590/S1807-55092013005000006

22. Denadai BS. Aspectos fisiológicos relacionados com a economia de movimento. Rev Bras Ativ Fís Saúde 1996;1(3):59-73. doi: 10.12820/rbafs.v.1n3p59-73

23. Wu SSX, Peiffer JJ. Pacing strategies during the swim, cycle and run disciplines of sprint, Olympic and half-Ironman triathlons. Eur J Appl Physiol 2015;115:1147-54. doi: 10.1007/s00421-014-3096-2

24. Bernard T, Vercruyssen F, Mazure C, Gorce P, Hausswirth C, Brisswalter J. Constant versus variable-intensity during cycling: effects on subsequent running performance. Eur J Appl Physiol 2007;99(2):103-11. doi: 10.1007/s00421-006-0321-7

25. Dalleau G, Belli A, Bourdin M, Lacour JR. The spring-mass model and the energy cost of treadmill running. Eur $\mathrm{J}$ Appl Physiol Occup Physiol 1998;77(3):257-63.

26. Borrani F, Candau R, Perrey S, Millet GY, Millet GP, Rouillon JD. Does the Mechanical Work in Running Change during the V'O2 Slow Component? Med Sci Sports Exerc 2003;35(1):507. doi: $10.1097 / 00005768-200301000-00009$

27. De Lucca L, Melo SIL. Relação entre modificações cinemáticas da corrida e o tempo limite na vVO2máx. Rev. Bras. Cineantropom. Desempenho Hum 2012;14(4):428438. doi: $10.5007 / 1980-0037$

28. Kreider RB, Boone T, Thompson WR, Burkes S, Cortes CW. Cardiovascular and thermal responses of triathlon performance. Med Sci Sports Exerc 1988;20(4):385-90.

29. Guezennec $C Y$, Vallier JM, Bigard AX, Durey A. Increase in energy cost of running at the end of a triathlon. Eur J Appl Physiol Occup Physiol 1996;73(5):440-5.

30. Hue O, Le Gallais D, Boussana A, Chollet D, Prefaut C. Ventilatory responses during experimental cycle-run transition in triathletes. Med Sci Sports Exerc 1999;31(10):1422-8.

31. Miura H, Kitagawa K, Ishiko T. Characteristic feature of oxygen cost at simulated laboratory triathlon test in trained triathletes. J Sports Med Phys Fitness 1999;39(2):101-6.

32. Peiffer J, Abbin CR, Sultana F, Bernard T, Brisswalter J. Comparison of the influence of age on cycling efficiency and the energy cost of running in well-trained triathletes. Eur J Appl Physiol 2016;116:195-201. doi: 10.1007/s00421-015-3264-z

33. Weltman A, Regan JD. Prior exhaustive exercise and subsequent, maximal constant load exercise performance. Int J Sports Med 1983;4(3):184-9.

34. Del Coso J, Gonzalez C, Abian-Vicen J, Martín JJS, Soriano L, Areces F, Ruiz D, Gallo C, Lara B, González JC. Relationship between physiological parameters and 
performance during a half-ironman triathlon in the heat. Journal of Sports Sciences 2014;32(18):1680-1687, doi: 10.1080/02640414.2014.915425

35. Cipriani DJ, Swartz JD, Hodgson CM. Triathlon and the multisport athlete. J Orthop Sports Phys Ther 1998;27(1):42-50.

36. Silva SRD. Fraga CHW, Gonçalves M. Efeito da fadiga muscular na biomecânica da corrida: uma revisão. Rev Motriz 2007;13(3):225-35.

37. Bonacci J, Saunders PU, Alexander M, Blanch P, Vicenzino $B$. Neuromuscular control and running economy is preserved in elite international triathletes after cycling. Sports Biomech 2011;10(1):59-71. doi: 10.1080/14763141.2010.547593

38. Cavagna GA, Kaneko M. Mechanical work and efficiency in level walking and running. J Physiol 1977;268(2):467-81. doi: 10.1113/jphysiol.1977.sp011866

39. Walsh JA, Stamenkovic A, Lepers R, Peoples G, Staply PJ. Neuromuscular and physiological variables evolve independently when running immediately after cycling. Journal of Electromyography and Kinesiology 2015;25(6):887-93. doi: 10.1016/j.jelekin.2015.10.008

40. McHardy A, Pollard H, Fernandez M. Triathlon injuries: A review of the literature and discussion of potential injury mechanisms. Clinical Chiropractic 2006;9:129-38. doi: 10.1016/j.clch.2006.04.001

41. Cosca DD, Navazio F. Common problems in endurance athletes. Am Fam Physician 2007;15;76(2):237-244.

42. Whiting W. C.; Zernicke R. F. Biomecânica funcional e das lesões musculoesqueléticas, v. 2. Rio de Janeiro: Guanabara Koogan, 2009.

43. Tuite MJ. Imaging of Triathlon Injuries. Radiol Clin North Am 2010;48(6):1125-35

44. Bonacci J, Chapman A, Blanch P, Vicenzino B. Neuromuscular adaptations to training, injury and passive interventions. Sports Med 2009;39(11):903-21. doi: 10.2165/11317850000000000-00000.

45. Le Meur Y, Thierry B, Rabita G, Dorel S, Honnorat G,
Brisswalter J, et al. Spring-Mass Behaviour during the Run of an International Triathlon Competition. Int J Sports Med 2013;34(8):748-55. doi: 10.1055/s-0032-1331205

46. Gottschall JS, Palmer BM. The acute effects of prior cycling cadence on running performance and kinematics. Med Sci Sports Exerc 2002;34(9):1518-22.

47. Millet GP, Bentley DJ. The physiological responses to running after cycling in elite junior and senior triathletes. Int. J. Sports Med 2004;25:191-97. doi: 10.1055/s-2003-45259

48. Girard O, Millet GP, Slawinski J, Racinais S, Micallef JP. Changes in Running Mechanics and Spring-Mass Behaviour during a 5-km Time Trial. Int J Sports Med 2013;34(9):83240. doi: 10.1055/s-0032-1329958

49. Bisi MC, Ceccarelli M, Riva F, Stagni R. Biomechanical and metabolic responses to seat-tube angle variation during cycling in tri-athletes. J Electromyogr Kinesiol 2012; 22(6):845-51. doi: 10.1016/j.jelekin.2012.04.013

50. Fletcher JR, Esau SP, Maclntosh BR. Economy of running: beyond the measurement of oxygen uptake. J Appl Physiol 2009;107(6):1918-22.

51. Vernillo G, Millet GP, Millet G. Does the running economy really increase after ultra-marathons? Front Physiol 2017; no prelo. doi: 10.3389/fphys.2017.00783

52. Cavagna GA. The landing-take-off asymmetry in human running. J Exp Biol 2006;209(20):4051-60.

53. Cavagna GA, Legramandi MA, Peyré-Tartaruga LA. The landing-take-off asymmetry of human running is enhanced in old age. J Exp Biol 2008;211(10):1571-8.

54. Hoff J, Støren Ø, Finstad A, Wang E, Helgerud J. Increased blood lactate level deteriorates running economy in world class endurance athletes. J Strength Cond Res 2016;30(5):1373-8.

55. Cavagna G. External, Internal and Total Mechanical Work Done During Locomotion. In Physiological Aspects of Legged Terrestrial Locomotion (pp. 129-138). Springer International Publishing. 2017.

Como citar: ONGARATTO, Daniela et al. Características fisiológicas e biomecânicas na corrida do triatlo: uma revisão narrativa. Cinergis, Santa Cruz do Sul, v. 18, n. 4, out. 2017. ISSN 2177-4005. Disponível em: < https://online.unisc.br/seer/index.php/cinergis/ article/view/8601 >. Acesso em: 07 dez. 2017. doi:http://dx.doi.org/10.17058/cinergis.v18i4.8601. 